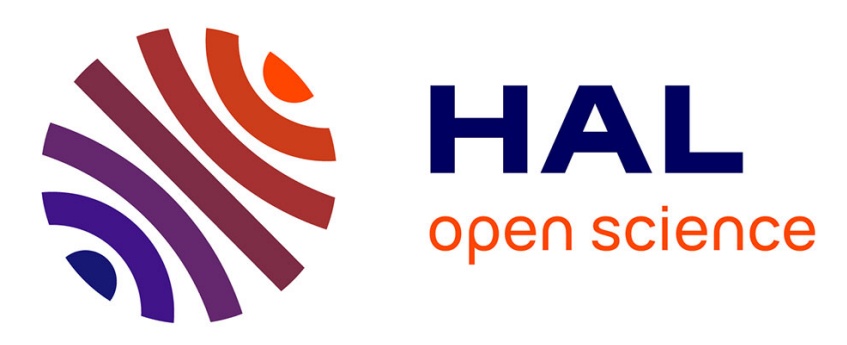

\title{
Numerical study of hydrodynamic impact on bubbly water
}

Mehdi Elhimer, Aboulghit El Malki Alaoui, Kilian Croci, Céline Gabillet, Nicolas Jacques

\section{- To cite this version:}

Mehdi Elhimer, Aboulghit El Malki Alaoui, Kilian Croci, Céline Gabillet, Nicolas Jacques. Numerical study of hydrodynamic impact on bubbly water. 34th International Conference on Ocean, Offshore and Arctic Engineering OMAE 2015, May 2015, St. John's, Canada. pp.OMAE2015-41889. hal01139860

\section{HAL Id: hal-01139860 \\ https://hal.science/hal-01139860}

Submitted on 7 Apr 2015

HAL is a multi-disciplinary open access archive for the deposit and dissemination of scientific research documents, whether they are published or not. The documents may come from teaching and research institutions in France or abroad, or from public or private research centers.
L'archive ouverte pluridisciplinaire HAL, est destinée au dépôt et à la diffusion de documents scientifiques de niveau recherche, publiés ou non, émanant des établissements d'enseignement et de recherche français ou étrangers, des laboratoires publics ou privés. 


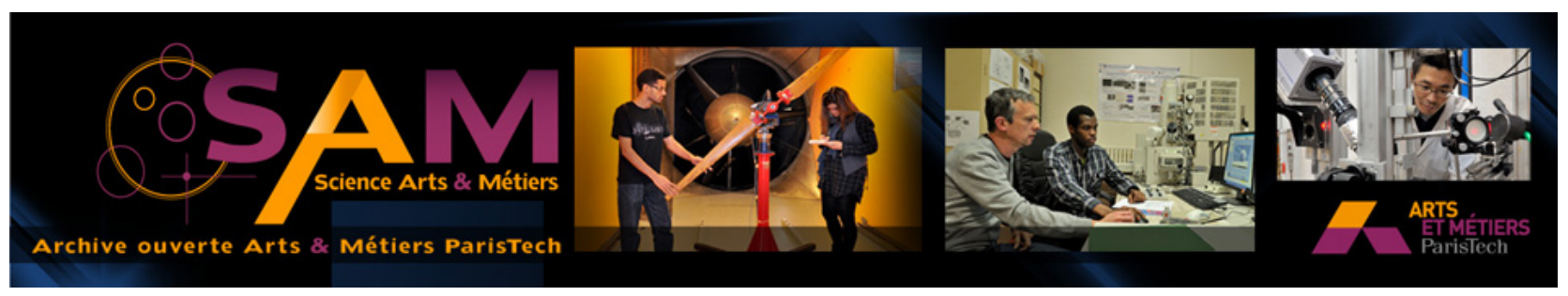

\section{Science Arts \& Métiers (SAM)}

is an open access repository that collects the work of Arts et Métiers ParisTech researchers and makes it freely available over the web where possible.

This is an author-deposited version published in: http://sam.ensam.eu

Handle ID: .http://hdl.handle.net/10985/9438

\section{To cite this version :}

Mehdi ELHIMER, Aboulghit EL MALKI ALAOUI, Kilian CROCI, Céline GABILLET, Nicolas JACQUES - Numerical study of hydrodynamic impact on bubbly water - In: 34th International Conference on Ocean, Offshore and Arctic Engineering OMAE 2015, Canada, 2015-05-31 Proceedings of the ASME 2015 34th International Conference on Ocean, Offshore and Arctic Engineering OMAE2015 May 31-June 5, 2015, St. John's, Newfoundland, Canada - 2015 


\section{NUMERICAL STUDY OF HYDRODYNAMIC IMPACT ON BUBBLY WATER}

\author{
Mehdi ELHIMER \\ ENSTA Bretagne \\ Brest, France
}

\author{
Céline GABILLET \\ Ecole Navale \\ Lanvéoc, France
}

\author{
Aboulghit EL MALKI ALAOUI \\ ENSTA Bretagne \\ Brest, France
}

\author{
Kilian CROCI \\ ENSTA Bretagne \\ Brest, France
}

\author{
Nicolas JACQUES \\ ENSTA Bretagne \\ Brest, France
}

\section{ABSTRACT}

The phenomenon of slamming on a bubbly liquid has many occurrences in marine and costal engineering. However, experimental or numerical data on the effect of the presence of gas bubbles within the liquid on the impact loads are scarce and the related physical mechanisms are poorly understood. The aim of the present paper is to study numerically the relationship between the void volume fraction and the impact loads. For that purpose, numerical simulations of the impact of a cone on bubbly water have been performed using the finite element code ABAQUS/Explicit. The present results show the diminution of the impact loads with the increase of the void fraction. This effect appears to be related to the high compressibility of the liquid-gas mixture.

\section{INTRODUCTION}

The design of naval and offshore structures requires the consideration of water impact related loads. An example of water impact problem is the slamming of a ship's bow in heavy sea conditions. Slamming loads can induce important transient stresses and affect locally and globally the structural integrity of the ship's hull.

The specific problem of a structure impacting on a bubbly fluid is found in several engineering situations. This configuration is encountered when computing the loads applied on a solid wall when submitted to the impact of a mass of liquid containing bubbles. Examples of this flow are the violent sloshing in LNG carriers [1] and the impact of breaking waves on costal structures [2].

The complexity of the problem came from the need of taking into account the multiphasic nature of the flow. It is well known that the compressibility of a liquid gas mixture is higher than that of a pure liquid. This is due to the higher compressibility of the gaseous phase in comparison to that of the liquid.

In addition the sonic speed decreases rapidly with the increase of the gas volume faction (or void fraction). Therefore for blunt solid impacts, the expansion velocity of the wetted surface reach supersonic values even for moderate impact velocities $[3,4]$. Classical impact theories consider only the case of incompressible fluid [5]. The need to account for the effects of the mixture compressibility in the computation of the impact loads has been highlighted by several authors. Skalak and Feit [6] and Korobkin [7] have extended these theories to the case of a compressible fluid using the acoustic approximation. This approximation is however of limited practical interest since its permits only small perturbation of the mixture density around its initial value,

Smaller number of works have been dedicated to the specific case of impact on a bubbly liquid [8-10]. These works show that the aeration affects considerably the effects both the loads on the solid and the fluid flow around it during the impact. This is often called the "cushioning effect" of the air bubbles.

Data from experiments and numerical simulations are still scarce in the literature. To our best knowledge, impact experiments on bubbly water with precisely controlled void fraction have only been performed by Eroshin [11].

The present work is included in a project aimed to quantify the effects of the aeration on the loads and pressures on impacting structures. To that purpose, we undertake numerical and experimental study of the impact of a rigid solid of simple geometrical form upon the surface of a two-phase mixture with an initially homogenous gas void fraction. 


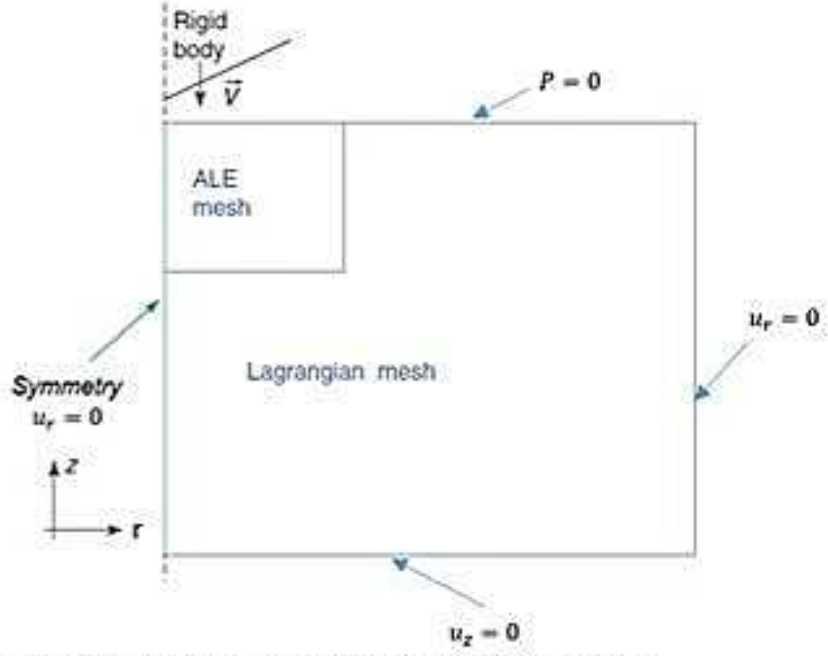

Figure 1 : scheme of the computational model

In this communication, we report results from numerical simulations performed using the $\mathrm{ABAQUS}$ /Explicit finite elements code. We simulated the impact of a cone on the surface of a two phase air-water mixlure, where the initial air void fraction is homogeneous all over the computation domain. The mixture response is calculated by the code using an a priori defined equation of state which will be presented in the following.

\section{NUMERICAL SIMULATIONS}

$A B A Q U S$ is a suite of simulation programs based on the finite elements method. The specific module ABAQUS/Explicit is suitable for modeling dynamic events of short duration, such as slamming. For that purpose, it uses an explicit and dynamic finite-elements formulation.

Using ABAQUSiExplicit, we have performed numerical simulations of the impact of a cone on the free surface of an airwater mixture. The mixture is represented as an equivalent homogenous fluid, characterized by its equivalent density and equation of state. The impacting solid considered in these simulations is a rigid, axisymmetric cone. Its dead rise angle is set to $\beta=7^{\circ}$ and its base's radius is set to $165 \mathrm{~mm}$. The fluid domain is also considered as axisymmetric. Normal velocity is set to zero on the fluid domain boundaries, except the free surface.

ABAQUS uses by default a Lagrangian approach, where the mesh moves with the fluid motion. The drawback of the Lagrangian framework in the present case is that large deformations of the fluid take place near the impact region, leading 10 an excessive distortion of the mesh elements. In order to avoid this problem, the adaptive meshing technique of ABAQUS was employed. This method is based on an arbitrary Euler-Lagrange (ALE) formalism, in which the mesh moves independently of the fluid flow. In order to reduce the computational cost of the simulations, the ALE adaptive meshing technique is used only for the impact region, where large deformations are expected to occur. Elsewhere, a Lagrangian meshing is used as shown in Figure 1.

This ALE region is finely meshed using 110249 axisymmetric 4 node elements with reduced-integration (ABAQUS CAX4R). All these elements having the same size. The rest the domain is meshed with 68395 axisymmetric 3node linear elements (CAX3). These elements size increase with the distance to the ALE region. The size of the computational domain was set to be sufficiently large, in order to avoid the influence of the acoustic wave reflections on the domain's boundaries.

For modelling the contact between the solid and the fluid surfaces, we use regularized contact law which allow the contact pressure at the nodes to increase continuously as the two bodies get closer. The ability of the ABAQUS code to accurately predict slamming loads was checked in the case of an homogeneous liquid through comparisons with theoretical and experimental results of the literature, see $[12,13]$. in the case of a blunt cone, Jacques et al report [12] a good agreement on pressure and impact force with experiments and CFD results.

\section{EQUATION OF STATE OF THE AIR-WATER MIXTURE}

In this section, the equation of state of the air-water mixture will be detined. For that purpose let us consider an elementary volume $V$ of this mixture. The initial volume of each of the water and the air are written $V_{L}$ and $V_{G}$ respectively, such that $V=V_{L}+$ $V_{G}$. The initial values of the volumes are written $V_{G 0}, V_{l .0}$ and $V_{0}$, and the initial void fraction is written $\alpha_{D}$. The air density is negligcable in comparaison with the water density. The density of the two phases mixture is:

$$
\rho_{0}=\rho_{L 0}\left(1-\alpha_{0}\right) \quad(1)
$$

Where $\rho_{L 0}$ is the initial density of the water.

Both the gas and the liquid phases are considered as invicid and barotropic media. The gaz and liquid pressure are written $P_{G}$ and $P_{L}$ respectively. The effect of the surface tension is neglected and we assume that the representative volume is in static equilibrium. Therefore, the pressure is the same for the two phases: $P_{L}=$ $P_{G}=P$.

With these hypotheses, the air water mixture can be regarded as a barotropic fluid. It has with a unique presssure-density relationship. It should be noted that the individual dymamics of the bubbles are not taken into account. Thus, we consider that the bubbles react instantaneously to a pressure change. An investigation of the influence of bubble dynamics in slamming events is under progess. In the present communication only results derived from the barotropic model are presented.

It can be easily shown that the variation of the elementary volume is related to the variation of the volume of each of the two phases by the equation :

$\frac{v}{v_{0}}=\alpha_{0} \frac{v_{G}}{v_{G 0}}+\left(1-\alpha_{0}\right) \frac{v_{L}}{v_{L 0}}$

Each term of this equation can be related to the local pressure $P$ in the mixture by using the equation of state of each phase. The air is considered as a perfect gas, using the Laplace law leads to the equation of state : $V_{G} / V_{G 0}=\left(P / p_{0}\right)^{-1 / \gamma}$ where $\gamma$ is the Laplace coefficient. For small enough bubbles ( with size less 
than $1 \mathrm{~mm}$ ), mechanical equilibrium is reached within few microsecondes after a compression due to the impact. This time laps is generally smaller than the duration of the impact. Thus this compression is considered as isothermal so the Laplace coefficient was set to $\gamma=1$ in our simulations. A linear equation of state was used for the water $P-P_{0}=$ $-K_{L 0} \cdot\left(V_{L}-V_{L O}\right) / V_{L 0}$ where $K_{L D}$ is the intiale compressibility modulus of the liquid $\left(K_{L 0}=2.19 \mathrm{GPa}\right.$ for the water). Finally we obtain the following equation of state for the two phase mixture:

$$
\frac{V}{V_{0}}=\alpha_{0}\left(\frac{P}{P_{0}}\right)^{-\frac{1}{\gamma}}+\left(1-\alpha_{0}\right)\left(1-\frac{P-P_{0}}{K_{L 0}}\right)
$$

\section{HYDRODYNAMIC IMPACT THEORIES}

\section{Dimensional analysis}

Let recall the configuration under study: We consider a rigid cone with a deadrise angle $\beta$ impacting upon the free surface of a liquid. The cone is axisymmetric around the vertical axis $\vec{z}$ and its impact velocity is $\vec{V}=-V_{i} \cdot \vec{z}$. The fluid is initially at rest, filling the domain $z<0$ and its free surface is perpendicular to the $\mathrm{z}$ axis(Figure 2).

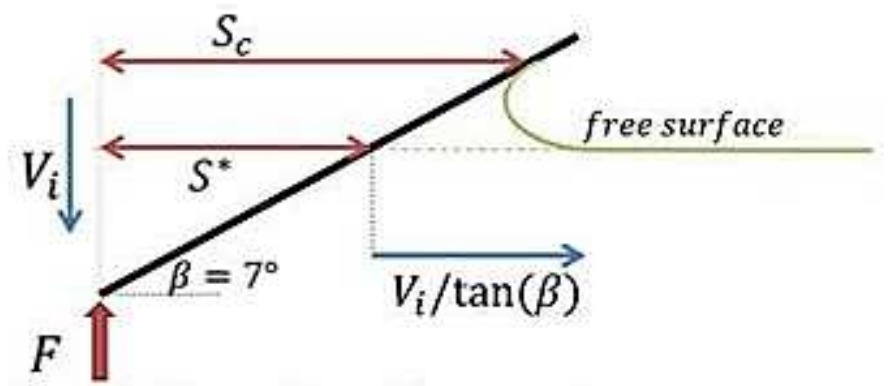

Figure 2 scheme of the problem geometry

The initial density of the fluid is written $\rho_{0}$. The effects of viscosity, gravity and surface tension are neglected. From dimensionnal analysis the following expression of the total hydrodynamic force on the cone $F$ :

$F=k \rho_{0} V_{i}^{4} t^{2}$

Where appears the (non-dimensional) slamming coefficient $k$. If the fluid is incompressible this coefficient depends only on the cone dead-rise angle : $k=k(\beta)$. For a compressible fluid $k$ is also dependent on the physical parameters related to the fluid compressibility and to the impact velocity: $k=k\left(\beta, P_{0}\right)$ $\left.\left(\rho_{0} V_{i}^{2}\right), K_{L 0} /\left(\rho_{0} V_{i}^{2}\right), \alpha_{0}, \gamma\right)$.

In the following, we use the non-dimensional slamming coefficient $f$ previously introduced by El Malki Alaoui [14].

$f=k \cdot \tan ^{3}(\beta)=\frac{F \tan ^{3}(\beta)}{\rho_{0} v_{i}^{4} t^{2}}$

For an incompressible liquid and a cone with a deadrise angle of $\beta=7^{\circ}$ we have $f=7.18$. This parameter will be used later to discuss the effect of the compressibility on the hydrodynamic loads.

\section{Acoustic approximation for supersonic impacts}

An analytical model for the impact on a compressible fluid have first been developed by Skalak and Feit [6]. This model is based on the acoustic theory. Let us denote the speed of sound in the fluid by $c$. This model is applicable for any impact velocity. A simple analytical expression for the impact load is available only in the supersonic impact regime. This regime occurs when the expansion velocity of the wetted surface exceeds the speed of sound in the fluid:

$$
\frac{V_{l}}{\tan (\beta)}>c
$$

In this case the pressure perturbation does not propagate beyond the wetted surface during the impact. Thus the free surface remains undisturbed. For this regime, Skalak and Feit have derived a simple expression for the impact force

$$
F=\rho_{0} c V_{i} A_{0}
$$

Where $A_{0}$ is the area of the projection of the wetted surface on the plane $z=0$, and $c$ is the sonic speed in the fluid. For a cone this area is $A_{0}=\pi\left(V_{i} t / \tan (\beta)\right)^{2}$. From the equation (7), the average pressure defined as $P_{m}=F / A_{0}$ can be expressed as $P_{m}=\rho_{0} c V_{l}$ leading to the equation:

$$
\frac{P_{m}}{\rho_{0} V_{i}}=c
$$

Thus in the supersonic regime, the ratio $P_{m} / \rho_{0} V_{i}$ is independant from the impact velocity and equal to the sonic speed $c$ in the fluid. The speed of sound in a bubbly liquid can be obtained using Wood's [15] formula:

$$
\begin{array}{r}
c=\left[\frac{1}{c_{G}^{2}}\left(\alpha_{0}^{2}+\alpha_{0}\left(1-\alpha_{0}\right) \frac{\rho_{L}}{\rho_{G}}\right)+\frac{1}{c_{L}^{2}}\left(\left(1-\alpha_{0}\right)^{2}+\right.\right. \\
\left.\left.\alpha_{0}\left(1-\alpha_{0}\right) \frac{\rho_{G}}{\rho_{L}}\right)\right]^{-1 / 2} \text { (9) }
\end{array}
$$

Where $c_{G}$ and $c_{L}$ are the sonic speed in air and water respectively. From this formula it can be shown that the sonic speed in bubbly flow decreases rapidely with the increase of the void fraction $\alpha_{0}$. It is equal to $c=1497 \mathrm{~m} / \mathrm{s}$ for pure water and drops to $c=$ $100 \mathrm{~m} / \mathrm{s}$ for $c_{0}=1 \%$ and $c=46 \mathrm{~m} / \mathrm{s}$ for $\alpha_{0}=5 \%$. Thus, for a cone with $\beta=7^{\circ}$ the supersonic regime may be reached with moderated impact velocities such as $V_{i}=12.3 \mathrm{~m} / \mathrm{s}$ for $\alpha_{0}=$ $1 \%$ and $V_{i}=5.6 \mathrm{~m} / \mathrm{s}$ for $\alpha_{0}=5 \%$.

However, it will be shown further that the supersonic regime is observed for significantly larger velocities.

\section{RESULTS AND DISCUSSION}

\section{Dimensional analysis}

First let us introduce few useful definitions. The ensemble of the mesh nodes in contact with the cone defines the contact surface and its area is noted $S_{c}$. The sum of the contact forces over all the nodes of the contact surface defines the impact fore $F$. The 
average pressure on the contact surface is defined as $P_{m}=$ $F /\left(S_{c} \cdot \cos (\beta)\right)$.

In order to cover a large range of impact regimes, the inpact velocity $V_{i}$ was varied from $V_{i}=0.2 \mathrm{~m} / \mathrm{s}$ to $V_{i}=700 \mathrm{~m} / \mathrm{s}$. Thus, the theoretical wetted surface expansion velocity $V_{i} / \tan (\beta)$ ranges between $1.6 \mathrm{~m} / \mathrm{s}$ and $5700 \mathrm{~m} / \mathrm{s}$.

The values of the initial void fractions considered in our simulations are $\alpha=1 \%, \alpha=5 \%$ and $\alpha=10 \%$ in addition to the case of pure water $\alpha=0 \%$. As it will be shown further, these void fractions are large enough to induce a significant effect on the impact loads and pressures.

\section{Effects of the aeration on the impact loads}

The aim of our simulations is to highlight and quantify the effect of the aeration on the impact loads. For that purpose we have compared the impact force $F_{\alpha}$ for each void fraction $\alpha$ to the impact force $F_{0}$ for the pure liquid at the same impact velocity $V_{i}$ and the same penetration depth. In Figure 3 we present the ratio $F_{c k} / F_{0}$ as a function of the expansion velocity $V_{i} / \tan (\beta)$.

It is clearly shown that the acration causes a decrease of the impact load. Indeed, we notice that $F_{\alpha} / F_{0}<1$ for any $\alpha \neq 0$ and for any impact velocity. Moreover, the impact load reduction increases with the volume fraction. For example, for an impact velocity of $V_{i}=30 \mathrm{~m} / \mathrm{s} F_{\alpha}$ drops of nearly $35 \%$ as the void fraction increases from $\alpha=1 \%$ to $\alpha=5 \%$.

The effect of the aeration also depends on the impact velocity. For the three values of $\alpha$ considered, the ratio $F_{\alpha} / F_{0}$ reaches its minimum value for an impact velocity of about $30 \mathrm{~m} / \mathrm{s}$ (the corresponding value of $V_{L} / \tan (\beta)$ is $245 \mathrm{~m} / \mathrm{s}$ ). The impact load reduction is smaller as $V_{i} \rightarrow 0$ or $V_{i} \rightarrow \infty$.

This asymptotic behavior may be explained as follows. $A s V_{i} \rightarrow$ 0 the effects of air compressibility and the related load reduction decrease significantly. For very large impact velocities the compressibility of water prevails, so the effect of air compressibility becomes negligible.

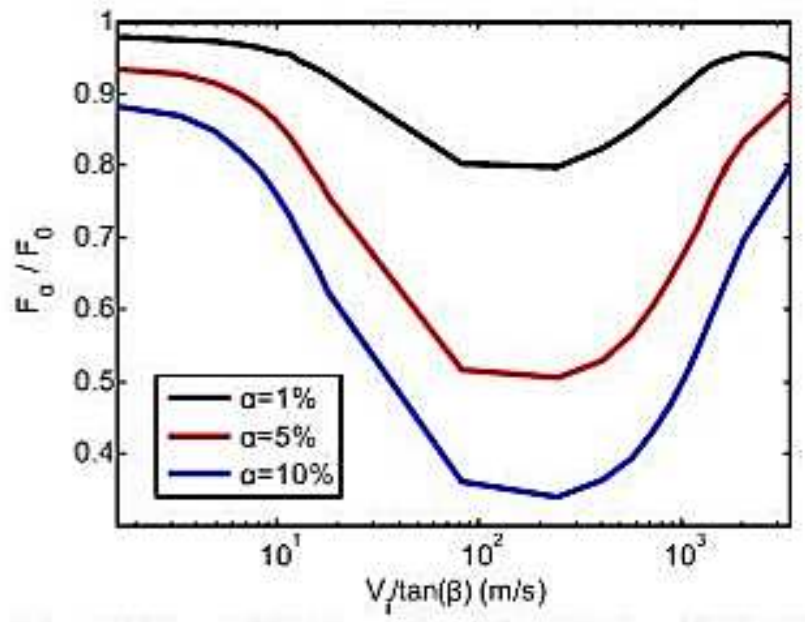

Figure 3 The ratio $F_{\alpha} / F$ as a function of $V_{i} / \tan (\beta)$ for bubbly water at three different initial void fractions $\alpha$
We now turn attention to the influence of the acration on the average pressure $P_{m}$ on the contact surface. We consider the ratio of the average pressure $P_{\alpha}$ for a void fraction $\alpha$ to the same pressure for pure water $P_{0}$. The evolution of $P_{\alpha} / P_{0}$ with $V_{i} / \tan (\beta)$ is shown in Figure 4 . We notice that for a given $V_{i}$ and $\alpha$, the reduction of the pressure $P_{m}$ is smaller than for the impact force $F_{\alpha}$.

This is due to the fact that the contact surface is modified by the aeration, as shown in Figure 5. It highlights a reduction of the contact surface area with the increase of the expansion velocity up to $V i / \tan (\beta)=1500 \mathrm{~m} / \mathrm{s}$. For larger velocities $S_{c}$ is constant for any void fraction. This is indicative of the appearance the supersonic impact regime, where the free surface is undisturbed during the impact.

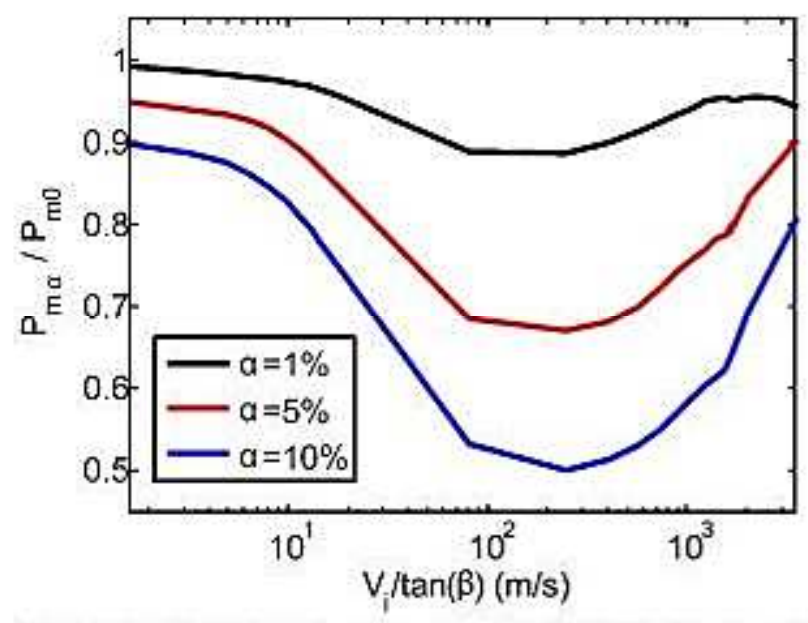

Figure 4 The ratio $P_{m a} / P_{m}$ as a function of $V_{i} / \tan (\beta)$ for bubbly water at three different initial void fractions $\alpha$

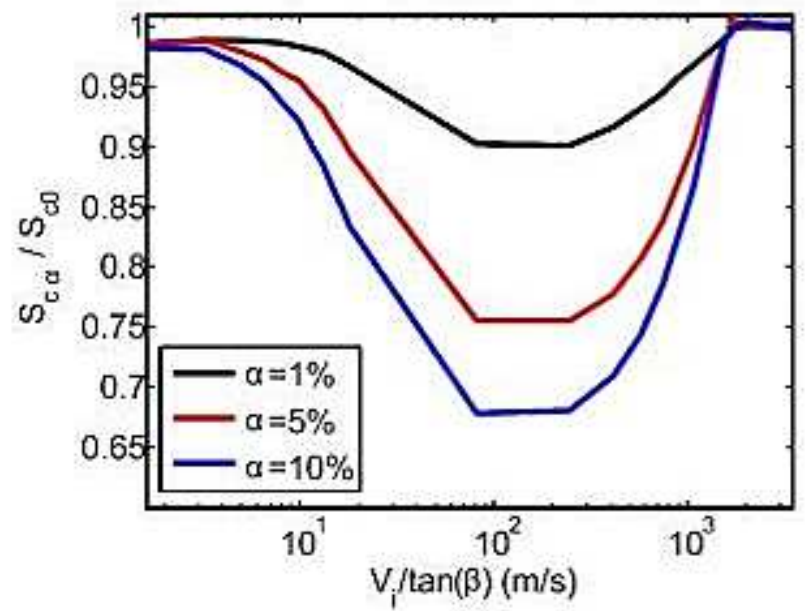

Figure 5 the ratio $s_{c \alpha} / S_{c 0}$ as a function of $V_{i} / \tan (\beta)$ for bubbly water at three different initial void fractions $\alpha$ 


\section{Effect of the air compressibility}

The effect of the air compressibility is investigated through the examination of the slamming coefficient $f$ (equation 5), the results are presented in Figure 6 . For an inconpressible fluid, this coeflicient is constant and equal to $f=7.18$ ( for $\beta=7^{\circ}$ ). For all void fractions the coefficient $f$ reached asymptotically this value when $V_{i} / \tan (\beta) \rightarrow 0$. However, $f$ decreases rapidly as $V_{i}$ increases when $\alpha$ is finite. This confirms that the previously observed load reduction is mainly due to the enhanced compressibility induced by the aeration. Besides, as $V_{t}$ increases the disparity between the values of $f$ for different void fractions is shown to decrease and even vanish for $V_{i} / \tan (\beta)>$ $3500 \mathrm{~m} / \mathrm{s}$. In fact, at the very high impact velocity, the influence of compressibility of the liquid phase is predominant and therefore the effect of the aeration becomes less pronounced.

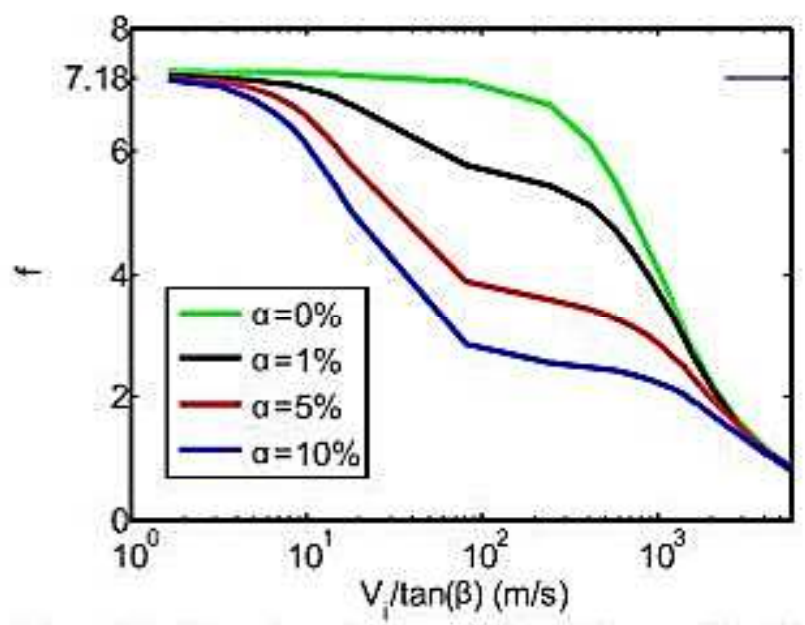

Figure 6 : The slamming coefficient $f$ as a function of $v_{i} / \tan (\beta)$ at three different initial void fractions $\alpha$ and for pure water $\alpha=0 \%$

\section{Supersonic impact regime}

A supersonic impact regime is expected to be observed for sufficiently large $V_{t}$. In this regime the free surface of the liquid remains undisturbed during the impact. Thus, the contact surface $S_{c}$ is equal to the surface $S^{*}$ of the cone under the initial free surface. The Figure 7 displays the evolution of the ratio $S_{c} / S^{*}$ with $V_{i} / \tan (\beta)$ for different void fractions. The supersonic impact regime occurs when this ratio becomes equal to 1 . We observe that this regime appears when $V_{i} / \tan (\beta)$ exceeds a critical value close to $1500 \mathrm{~m} / \mathrm{s}$. Unexpectedly, this critical velocity is the same for the three values of void fraction considered. It is close to the sonic speed in water, but differs from the sonic speed in bubbly water. This unforeseen is most likely linked to the nonlinearity of the equation of state of the airwater mixture. The impact involves a significant increase of the pressure close to the wetted surface. Thus the air is compressed and its volume diminish. As a result the local void fractions drops significantly, so the local sonic velocity increases towards its value in pure water.
Now, we compare our results to the modelling of Skalak and Feit based on the acoustic theory. As mentioned previously, this model predicts that the ratio $P_{m} / \rho_{0} V_{k}$ is equal to the speed of sound in the supersonic regime. In Figure 8 we represent the values of $P_{m} / \rho_{0} V_{l}$ from our simulations as a function of $V_{i} / \tan (\beta)$.

For pure water, a good agreement with the theoretical predictions is found. Indeed when $V_{i} / \tan (\beta)$ is larger than the sonic speed in water $c_{0}=1497 \mathrm{~m} / \mathrm{s}$ the ratio $P_{m} / \rho_{0} V_{i}$ is almost constant and close to $c_{0}$. In contrast, for large values of void volume fraction ( $\alpha=5 \%$ and $\alpha=10 \%$ ), the ratio $P_{m} / \rho_{0} V_{l}$ continue to increase substantially in the supersonic regime. Besides, the value of this ratio exceeds the speed of sound in the mixture and is in fact closer to the speed of sound in water.

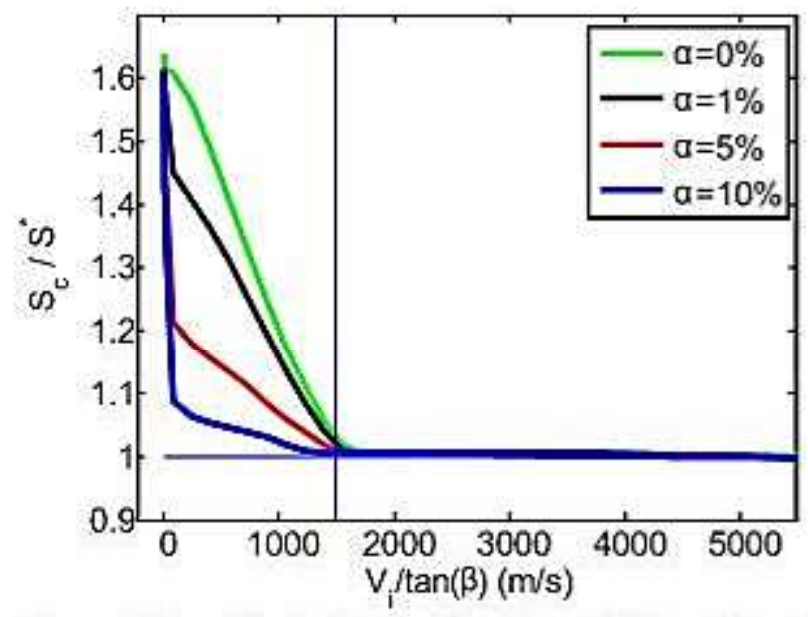

Figure 7 The ratio $S_{c} / S$ as a function of $V_{i} / \tan (\beta)$ at three different initial void fractions $\alpha$ and for pure water $\alpha=0 \%$

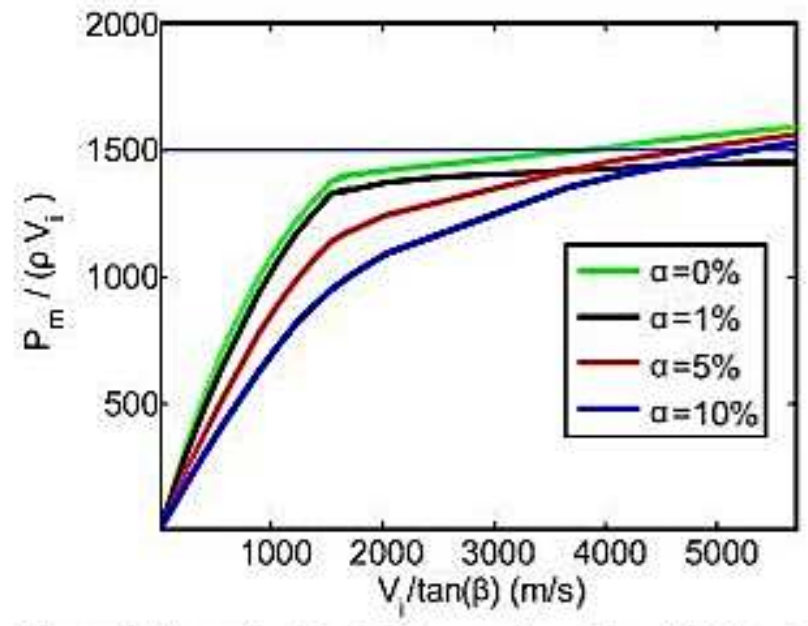

Figure 8 The ratio $P_{m} /\left(\rho V_{i}\right)$ as a function of $V_{i} / \tan (\beta)$ at three different initial void fractions $\alpha$ and for pure water $\alpha=$ $0 \%$ 


\section{CONCLUSIONS AND PERSPECTIVES}

In the present study, numerical simulations of the impact of a rigid cone on the surface of an aerated liquid have been performed. Different values of the initial void fraction and a large range of inpact velocities have been investigated. Our results show that the aeration reduces significantly the impact loads and affects the flow around the impacting body. This cushioning effect is attributed to the enhanced compressibility of the mixture in comparison to pure water.

A supersonic impact regime has been highlighted for all void fractions. Noteworthily, this regime appears when the expansion. velocity of the cone's wetted surface exceeds the sonic speed in pure water, and not in the air-water mixture. This observation needs to be further investigated.

As a perspective, we intend to continue the present study by considering impacting bodies with different geometries. In addition, the influence of bubbles dynamics phenomena on the impact loads will be investigated by employing the model from [16].

Impact experiments on bubbly water are scheduled and are currently under preparation. These experiments require a device capable of generating sufficiently small bubbles with a homogeneous void fraction. For that purpose, the use of an array of porous soaker hoses is a simple and cost-effective way to aerate a large volume of water [17].

\section{REFERENCES}

[1] Ten, I., and Korobkin, A., 2009, "Elastic response of vertical wall to aerated fluid impact," Hydroelasticity in Marine Technology, University of Southampton, UK. pp. 121-130.

[2] Bullock, G. N., Obhrai, C., Peregrine, D. H., and Bredmose, H., 2007, "Violent breaking wave impacts. Part 1: Results from large-scale regular wave tests on vertical and sloping walls," Coast. Eng, 54, pp. 602617.

[3] Kapsenberg, G. K., 2011, "Slamming of ships: where are we now?", Philos. Trans. A. Math. Phys. Eng. Sci., 369. pp. 2892-2919.

[4] Carcaterra, A., and Ciappi, E., 2000, "Prediction of the Compressible Stage Slamming Force on Rigid and Elastic Systems Impacting on the Water Surface," Nonlinear Dyn., 21, pp. 193-220.

[5] Wagner, H., 1932, "Uber Stoß- und Gleitvorgänge an der Oberfläche von Flüssigkeiten," J. Appl. Math.
Mech. / Zeitschrift für Angew. Math. und Mech., 12(4), pp. 193-215.

[6] Skalak, R., and Feit, D., 1966, "Impact on the Surface of a Compressible Fluid," J. Manuf. Sci. Eng, 88(3), pp. 325-331.

[7] Korobkin, A., 1994, "Blunt-body impact on the free surface of a compressible liquid," J. Fluid Mech., 263, pp. 319-342.

[8] Peregrine, D, H, and Thiais, L., 1996, "The effect of entrained air in violent water wave impacts,"J. Fluid Mech., 325, pp. 377-397.

[9] Bredmose, H., Peregrine, D. H., and Bullock, G. N., 2009 , "Violent breaking wave impacts. Part 2: modelling the effect of air," J. Fluid Mech., 641, pp. $389-430$.

[10] Dias, F., Dutykh, D., and Ghidaglia, J.-M., 2010, “A two-fluid model for violent aerated flows, "Comput. Fluids, 39, pp. 283-293.

[11] Eroshin, V. A., Romanenkov, N. I., Serebryakov, 1, V., and Yakimov, Y.L., 1980, "Hydrodynamic forces produced when blunt bodies strike the surface of a compressible fluide," Fluid Dyn., 15(6), pp. 829-835.

[12] Jacques, N., Constantinescu, A., Kerampran, S, and Nême, A., 2010, "Comparaison de différentes approaches pour la simulation numérique d'impacts hydrodynamiques," Eur. J. Comput. Mech. Eur. Mécanique Numérique, 19(8), pp. 743-770.

[13] Tassin, A., Jacques, N., El Malki Alaoui, A., Nême, A., and Leblé, B., 2010, "Assessment and comparison of several analytical models of water impact," Int. J. Multiphys., 4(2), pp. 125-140.

[14] El Malki Alaoui, A., Nême, A., Tassin, A., and Jacques, N., 2012, "Experimental study of coefficients during vertical water entry of axisymmetric rigid shapes at constant speeds," Appl, Ocean Res., 37, pp. 183-197.

[15] Wood, A., 1941, A textbook of sound, G. Bell and Sons Ltd., London.

[16] Grandjean, H., Jacques, N., and Zaleski, S., 2012, "Shock propagation in liquids containing bubbly clusters: a continum approach," J. Fluid Mech., 701, pp. 304-332.

[17] Giovannettone, J. P., Tsai, E., and Gulliver, J. S., 2009, "Gas void ratio and bubble diameter inside a deep airlift reactor," Chem. Eng. J., 149(I-3), pp. 301-310. 